\title{
Aerotecnica M\&S 100 Years Ago: A Study on Aircraft Longitudinal Stability
}

\author{
Preface to Issue 1, Volume 101
}

\author{
Aldo Frediani ${ }^{1} \cdot$ Vittorio Cipolla $^{1} \cdot$ Sergio De Rosa ${ }^{2} \cdot$ Paolo Gasbarri $^{3}$
}

Accepted: 7 February 2022 / Published online: 15 February 2022

(c) The Author(s) under exclusive licence to AIDAA Associazione Italiana di Aeronautica e Astronautica 2022

\section{On the Longitudinal Stability of Airplanes ${ }^{1}$}

\subsection{Relationship Between Static and Dynamic Stability in the Hypothesis of no Oscillations of the CG: Relative Measurement Method. Approximate Study of the Jolt}

\subsubsection{F. Burzio, Politecnico di Torino}

The general treatment of the longitudinal stability problem (which is the study of a rigid body in the plane of motion, composed of the motion of the Centre of Gravity (or "jolt" in the following) in a fixed vertical plane and of the rotation of the system (pitch) around an axis normal to the plane with the origin in the Centre of Gravity) does not reveal in a simple way the relationship existing between what is usually called static longitudinal stability, or initial, or intrinsic, and the dynamic phenomenon (dynamic stability, measured by certain parameters, which we will see later on in the paper) which is the consequence of statics and other factors (moment of inertia). Also with the obtained methods of measurement, we determine independently the static stability ${ }^{2}$ and the dynamic one, ${ }^{3}$ without the possibility to compare them. A simple relationship between the two quantities can be established with the assumption that the oscillation around the barycentric axis occurs without giving rise to alterations in the motion of the centre of gravity, that is, with the study of simple pitch, supposed the null jolt. The resulting difference is discussed in the following.

Aldo Frediani

aldo.frediani@unipi.it

Università di Pisa, Pisa, Italy

2 Università Federico II Napoli, Naples, Italy

3 Università La Sapienza Roma, Rome, Italy
The general procedure leads, for small oscillations ${ }^{4}$ to a system of three differential equations in the variables: $q=\mathrm{d} \theta / \mathrm{d} t$. (angular pitch speed), $u, v$ (velocity components of the $\mathrm{CG}$, in the direction of motion and in the normal one, respectively):

$$
\left\{\begin{array}{c}
\frac{\mathrm{d} u}{\mathrm{~d} t}+v_{0} q=-g \theta \cos \theta_{0}+u X_{u}+v X_{v}+q X_{q} \\
\frac{\mathrm{d} v}{\mathrm{~d} t}-u_{0} q=-g \theta \sin \theta_{0}+u Z_{u}+v Z_{v}+q Z_{q} \\
I \frac{\mathrm{d} q}{\mathrm{~d} t}=u M_{u}+v M_{v}+q M_{q},
\end{array}\right.
$$

where $I$ is the moment of inertia of the system around the pitch axis, $X_{u} X_{v} Z_{u} \ldots M_{q}$ are the incremental ratios (resistance derivatives in the original test) of the components $X$ and $Z$ of the thrust and of its moment $\mathrm{M}$ relevant ot the $\mathrm{CG}$ with respect to $u, v$ and $q$.

The integration of this system can be carried out by the Routh's method whose determinant gives, for the general integrals of the form

$\theta=\theta_{1} e^{\lambda_{1} t}+\theta_{2} e^{\lambda_{2} t}+\theta_{3} e^{\lambda_{3} t}+\theta_{4} e^{\lambda_{4} t}, \ldots$,

a 4 th degree equation in $\lambda$ :

$\lambda^{4}+A_{1} \lambda^{3}+B_{1} \lambda^{2}+C_{1} \lambda+D_{1}=0$

where the coefficients are functions of $X_{u} \ldots M_{q}$, and of the known quantities. The 4 roots of the characteristic equation in $\lambda$, in the most interesting case for stability, are of the type

$\left(\lambda+a_{1} \pm i b_{1}\right)\left(\lambda+a_{2} \pm i b_{2}\right)=0$,

\footnotetext{
1 Presented on July 15th, 1922 in Turin.

2 A. Rota, "Sulla stabilità longitudinale dei velivoli", Rendiconti I.S.A., Anno IX, Serie 2 . "Statical longitudinal stability of airplanes", NACA-TR-96, National Advisory Committee for Aeronautics, 1921.

${ }^{3}$ Reports of National Advisory Committee for Aeronautics, p. 330 and following, 1917.

4 Bairstow, L., "Applied Aerodynamics", p. 457 and following.
} 
i.e.: complex conjugate roots corresponding to two damped oscillations of the form

$\theta=\theta_{0} e^{-\mu t} \operatorname{sen}\left(\frac{2 \pi}{T} t\right)$

where the parameters $\mu$ and $\mathrm{T}$ are functions of $\mathrm{a}_{1}$ and $\mathrm{b}_{1}$ in the 1 st oscillation, of $a_{2}$ and $b_{2}$ in the 2 nd. These, or rather the roots of two equations of the 2 nd degree that are derived from the approximate decomposition of the equation of 4th degree into $\lambda$, (Bryan, Bairstow) are physically interpreted as two coexisting pitch oscillations: the 1st (short oscillation) occurs at small $T$ and great $\mu$, the 2 nd (phugoid oscillation) generally occurs at large $T$ and small $\mu$ : the latter, at high speeds, could become an aperiodic motion.

If we make the hypothesis of null jolt $(u=v=0)$, the system (1) is reduced to the 3rd equation, modified as follows:

$I \frac{d^{2} \theta}{d t^{2}}=q M_{q}=\delta M$,

and we find ${ }^{5}(5)$

$\delta M=A \frac{d \theta}{d t}+B \theta$

where $A$ is proportional to the damping torque (or rotational, or dynamic stabilizer), $B$ to the static stabilizer torque. ${ }^{6}$

Equation (2) gives rise (in the case, more interesting for stability, of negative roots of the characteristic equation, and taking the initial condition $\theta=0$ for $t=0$ into account), to the general solution:

$\theta=\theta_{0} e^{-\mu t} \operatorname{sen}(h t)$

where:

$\mu=\frac{A}{2 I} ; h^{2}=\frac{B}{I}-\mu^{2}$,

that is, $\mu$ and $h$ are real part and modulus of the imaginary part of the roots of the characteristic equation, respectively.

Therefore, neglecting the jolt is the same as assuming pitch consisting of a single damped oscillation instead of the two present in the general solution: and it will be interesting to study in what relationship is that with these.

Now, we observe that, because (as is well known) the period of oscillation is:

\footnotetext{
5 See, for example, Verduzio "Teoria del volo dell'aeroplano" (Theory of the flight of aircraft), p. 230 and following or De Villers, "La dynamique de l'avion", p. 215 and following.

${ }^{6}$ The relationship with the longitudinal stability index, $c$, given by A. Rota, is $B=Q V^{2} \delta c$, where $Q$ is the aircraft weight, $V$ the speed and $\delta$ the density.
}

$T=\frac{2 \pi}{h}=\frac{2 \pi}{\sqrt{B / I-h^{2}}}$,

it results:

$\frac{B}{I}=\frac{4 \pi^{2}}{T^{2}}+\mu^{2}$,

This is, therefore, the simple relationship sought between static and dynamic stability, the parameters of the latter being $\mu$ and $T$. Equation (3) tells us that a great dynamic stability (large $\mu$, small $T$ ) can be obtained with a large static stabilizing moment, and with a small moment of inertia, and indeed, that the dynamic stability is the ratio of static stability and the moment of inertia.

This result is achieved in the hypothesis of simple pitching, that is, of zero jolt. It will therefore be important to study, in second approximations, this motion of the jolt, which has been neglected so far. This is also because, in experimental research on stability into the wind tunnel, it is possible to obtain only the simple pitch, the speed of the center of gravity (the wind speed) remaining, evidently, unchanged.

Equation (3) also offers a way for the experimental determination of static stability as a function of the dynamics one. The relevant apparatuses (aerodynamic torsion pendulums) are used for tunnel tests on models and can be of various types. Let's see the base theory.

In these devices, in addition to the damping moment $A^{\prime}$ and the static stabilizer moment of the model, (having the meaning of their corresponding $A$ and $B$ defined above for the actual aircraft), two other active moments have to be considered:

- a first one proportional to the angular displacement, and which, for example, in the pendulum designed by prof. Panetti, ${ }^{7}$ has the expression: $j \frac{G}{l} \theta$, where $j$ is the polar moment of inertia of the bar, $l$ its length and $G$ the shear modulus of elasticity;

- a damping one, due to the passive drag in the pendulum, free of the model. As far as the nature of this moment is concerned, it is quite evident that it should not contain appreciable terms proportional to $\theta$, since the drag will not depend, in general, on the position of the aircraft: thus, neglecting, by approximation, the powers of higher than the first of $\frac{\mathrm{d} \theta}{\mathrm{d} t}$ (which would complicate the integra-

\footnotetext{
7 As Fig. 1 shows, it is a small steel bar, A, connected to the rigid model $\mathrm{M}$ and supporting an oscillating mass $\mathrm{D}$ provided with a moment of inertia I: from the amplitude and duration of the oscillations it is possible to determine the parameters $\mu$ and $\mathrm{T}$ (see also Burzio, "Un metodo per la determinazione della stabilità longitudinale dei velivoli" (A method for the assessment of longitudinal stability of aircraft), A.N.I.I., 1922).
} 


\section{PENDOLO DI TORSIONE AERODINAMICO}

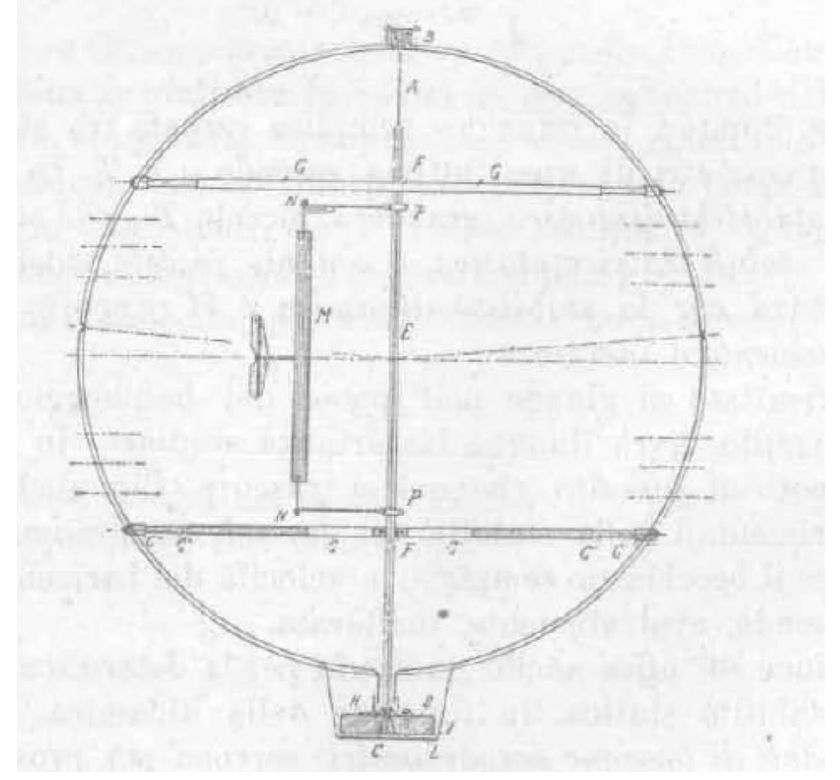

Fig. 1 Aerodynamic torsion pendulum

tion of the subsequent differential equations) ${ }^{(7)}$, this term will be of the type: $k \frac{\mathrm{d} \theta}{\mathrm{d} t}+c$, (where both $A^{\prime}$ and $k$ are of the type: $A^{\prime}=A^{\prime \prime} V+A^{\prime \prime \prime}, k=k^{\prime} V+k^{\prime \prime}$, respectively; furthermore, the apparatus, by means of tests carried out in still air and with wind, with and without model, allows also to determine separately the terms $A^{\prime \prime}$ and $A^{\prime \prime \prime}$, a $k$ and $k^{\prime \prime}$, that is, to separate the part due to the relative speed from that due to the simple rotation).

Then, it turns out, experimentally, that the constant $c$ is negligible, since, if it could not be zero, $c$ would assume the value $\frac{c}{{ }_{j} G_{/ l+B^{\prime}}}$ for $t=\infty$ : since, because $\theta$ becomes practically zero, it means that $c=0$

Thus, the differential equation of the aerodynamic torsion pendulum becomes:

$\left(I_{1}+I_{2}\right) \frac{\mathrm{d}^{2} \theta}{\mathrm{d} t^{2}}+\left(A^{\prime}+k\right) \frac{\mathrm{d} \theta}{\mathrm{d} t}+\left(B^{\prime}+j \frac{G}{l}\right) \theta=0$,

where $I_{1}$ and $I_{2}$ are the moments of inertia of the pendulum and of the model, respectively; (2'), in the case of complex roots of the characteristic equation, gives rise to the general integral:

$\theta=\theta_{0} e^{-\mu^{\prime} t} \operatorname{sen}\left(h^{\prime} t\right)$

where:
$\mu^{\prime}=\frac{A+k}{2\left(I_{1}+I_{2}\right)} ; h^{\prime 2}=\frac{j}{I_{1}+I_{2}} \frac{G}{I}+\frac{B^{\prime}}{I_{1}+I_{2}}-\mu^{\prime 2}$.

It results the following period:

$T^{\prime}=\frac{2 \pi}{\sqrt{\frac{j}{I_{1}+I_{2}} \frac{G}{I}+\frac{B^{\prime}}{I_{1}+I_{2}}-\mu^{\prime 2}}}$

Repeating the experiment without the model, we have:

$\left(I_{1}+I_{2}\right) \frac{\mathrm{d}^{2} \theta}{\mathrm{d} t^{2}}+k \frac{\mathrm{d} \theta}{\mathrm{d} t}+j \frac{G}{l} \theta=0$

with:

$\theta=\theta_{0} e^{-\mu_{0} t} \sin \left(h_{0} t\right)$,

where:

$\mu_{0}=\frac{k}{2\left(I_{1}+I_{2}\right)} ; T_{0}=\frac{2 \pi}{\sqrt{\frac{j}{I_{1}+I_{2}} \frac{G}{I}-\mu_{0}^{2}}}$.

From the expressions of $T^{\prime}$ and $T_{0}$, it results easily:

$\frac{B^{\prime}}{I_{1}+I_{2}}=\frac{4 \pi^{2}}{T^{\prime} 2}-\frac{4 \pi^{2}}{T_{0}^{2}}+\mu^{\prime} 2-\mu_{0}^{2}$

where the quantities $T^{\prime} T_{0} \mu^{\prime} \mu_{0}$ are immediately known from the experiment.

It is thus possible to determine experimentally $B^{\prime}$, proportional to the static stabilizing torque of the model.

According to the principles of mechanical similarity, and assuming equal densities, it will be $B=$ where $\gamma$ indicates the ratio of geometric similarity between the airplane and the model, and if $V$ and $V^{\prime}$ are the speed of the model and the real one, respectively.

Thus, for this method, the usual reserves for wind tunnel tests on models are valid as well: but note that we can carry out comparative evaluations between the qualities of static and dynamic stability of different aircraft, escaping the difficulty of mechanical similarity, by experimenting on models of the aircraft themselves, reduced to the same scale.

\section{An Approximate Study of the Jolt}

Assuming the initial equilibrium of the forces $Q$ (weight of the aircraft) $T$ (propeller traction force) $F$ (aerodynamic action), applied to the center of gravity $G$, a small amplitude pitch, $\theta$, has the following effects: $Q$ remains unchanged in intensity and direction; $T$ can be considered unchanged in magnitude, while it will rotate at the angle $\theta$, so that the new $T^{\prime}$ will be equal to the geometric sum of the $T$ with a force normal to $i t: \delta T=\sim T \theta$; the variation of wing incidence, 


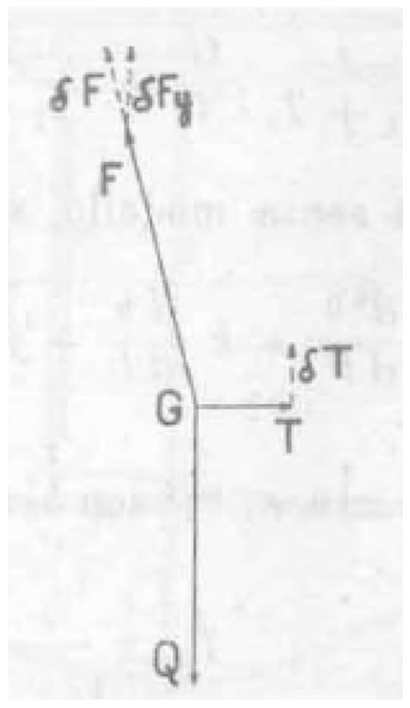

Fig. 2 (from the original paper)

da, will be given by the difference between the angle $\theta$ and the angular variation, $\varphi$, of the trajectory: but this is due only to the perturbation of the motion of the center of gravity, that is, to the jolt that we are studying, and it is not known until the same jolt is unknown: therefore, we refer to our fundamental scope to study the jolt as a consequence of the simple pitch, considering $\varphi=0$ in the first approximation, i.e.: $\delta \alpha=0$; finally the force $F$ will be varied in magnitude, and we can admit, by generality, in the direction of $\mathrm{d} F$, resulting from a $\delta F_{y}$ normal to the initial trajectory, and one, $\delta F_{x}$, opposite to the trajectory itself (Fig. 2).

Now we observe that, for aircraft with a good aerodynamic design, $\delta F_{x}$ is always very small compared to $\delta F_{y}$ so that we neglect it compared to the last one; moreover, considering, (it results for normal airfoils) that: $K_{y}=m+n a$, where $m$ and $n$ are constants, and a is the wing incidence, it will be: $F_{y}=(m+n \alpha) S V_{0}^{2}$ (if $S$ is the wing surface and $V_{0}$ the initial velocity) and, therefore $\delta F_{y}=n S V_{0}^{2} \delta \alpha=\sim n S V_{0}^{2} \theta$.

Thus, to the three initial forces $Q T F$ in equilibrium, the forces $\delta T$ and $\delta F \sim \delta F_{y}$ have been added: these two forces are responsible of the perturbation of the motion of the center of gravity, that is, the rise of a jolt speed: within the limits of the approximations indicated, we can consider the jolt motion as introduced by two forces both normal to the initial trajectory and proportional to $\theta$; i.e.:

$\delta F_{y}+\delta T=\left(n S V_{0}^{2}+T\right) \theta=\frac{Q}{g} \frac{\mathrm{d} v}{\mathrm{~d} t}$

if $v$ is the jolt speed: we study, so to say, the jolt as the motion of a point subject to a dampened classical force.

Putting:

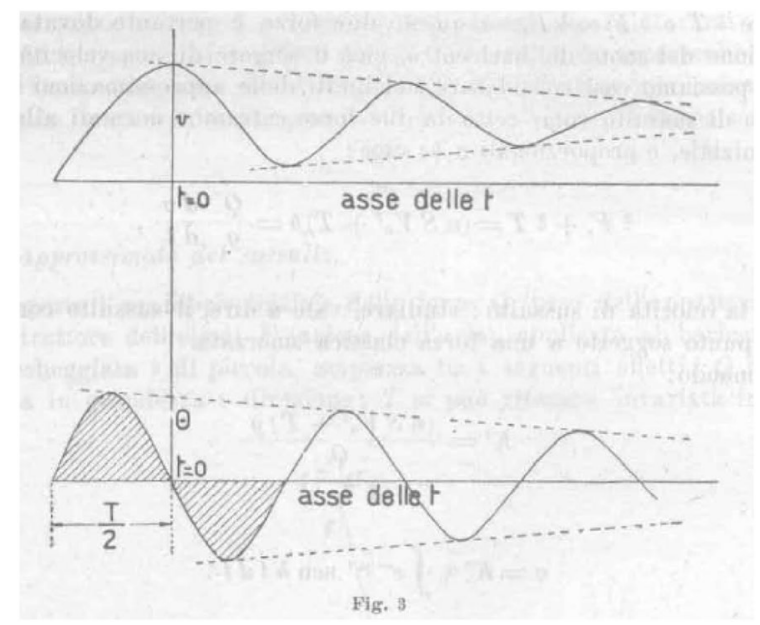

Fig. 3 (from the original paper)

$K^{\prime}=\frac{\left(n S V_{0}^{2}+T\right) g}{Q}$

it results:

$v=K^{\prime} \theta \int e^{-\mu t} \sin (h t) \mathrm{d} t$.

This $\int$ can be calculated by parts applied twice, exchanging the factors, and we have:

$\int e^{-\mu t} \sin (h t) \mathrm{d} t=\frac{h \mu}{h^{2}+\mu^{2}} e^{-\mu t}\left(\frac{\sin (h t)}{h}+\frac{\cos (h t)}{\mu}\right)+C$,

that is:

$v=K^{\prime} \theta_{0} \frac{h \mu}{h^{2}+\mu^{2}} e^{-\mu t}\left(\frac{\sin (h t)}{h}+\frac{\cos (h t)}{\mu}\right)+C$.

the value of $v(t=0)$, that is the initial value, depends on the disturb produced by the pitch, before $t=0$ : if, for simplicity's sake, we suppose e.g. that the disturb acts almost sinusoidally and with the same period of $\theta$, we will have:

$v_{0}=K^{\prime} \int_{-\frac{T}{2}}^{0} \theta \mathrm{d} t$

and considering the 1 st dotted area in Fig. 3 as a triangle with base $T / 2$ and height $\theta_{0}$, it will be ${ }^{8}$ :

$v_{0}=K^{\prime} \theta_{0} \frac{T}{4}$.

\footnotetext{
${ }^{8}$ Conversely, assuming the perturbation act almost instantaneously the expression would be $\$ \$\{\mathrm{v}\}_{-}\{0\}=\{\mathrm{K}\}^{\wedge}\left\{\mid \operatorname{text}\left\{{ }^{\prime}\right\}\right\}\{\backslash \text { theta }\}_{-}\{0\} \backslash$ frac $\{\mathrm{T}\}\{8\} \$ \$$
} 
So, we determine the value of constant $C$ :

$C=K^{\prime} \theta_{0}\left(\frac{T}{4}-\frac{h}{h^{2}+\mu^{2}}\right)$.

It results:

$v=K^{\prime} \theta_{0}\left[\left(\frac{T}{4}-\frac{h}{h^{2}+\mu^{2}}\right)+\frac{h \mu}{h^{2}+\mu^{2}} e^{-\mu t}\left(\frac{\sin (h t)}{h}+\frac{\cos (h t)}{\mu}\right)\right]$.

Or, by putting $\frac{\mu}{h}=\tan \psi$ :

$v=K^{\prime} \theta_{0}\left[\left(\frac{T}{4}-\frac{h}{h^{2}+\mu^{2}}\right)+\frac{h \mu}{h^{2}+\mu^{2}} e^{-\mu t} \frac{\cos (h t-\psi)}{\cos \psi}\right]$.

Equation (4) written for: $t=\infty$ shows that the jolt speed tends to a non-zero limit value, proportional directly to the initial pitch, inversely to the weight of the aircraft; it also depends on the mode of the disturbing cause and on the characteristics of static and dynamic stability of the aircraft.

Within the limits of the approximations, it would be concluded that the dampened elastic forces acting on the center of gravity because the pitch tends to modify the jolt speed from the initial value $v_{0}$ to a less final value but not zero. The extinction of this residual living force will be due to the passive drag of the aircraft in which the jolt motion takes place, drag that we have not considered because, being proportional $v^{2}$, the introduction into the differential equation of the jolt would have made it impossible its integration.

Finally, we can evaluate by approximation the effect of the jolt on the pitch, that is, what is neglected by studying the simple pitch.

Just remember pitch equation (the $3 \mathrm{rd}$ of the general system) (1):

$I \frac{\mathrm{d}^{2} \theta}{\mathrm{d} t^{2}}=d M$

and since, by assuming the barycentric traction force:
$M=\chi \mathrm{SV}^{2} a=\sim \chi_{y} S V^{2} a$,

if $a$ it is the arm of $\mathrm{F}$, it turns out:

$\mathrm{d} M=\frac{\partial \chi_{y}}{\partial M} \mathrm{~d} \chi_{y}+\frac{\partial M}{\partial V} \mathrm{~d} V+\frac{\partial M}{\partial a} \mathrm{~d} a$.

The simple pitch considers only the following:

$I \frac{\mathrm{d}^{2} \theta}{\mathrm{d} t^{2}}=\frac{\partial \chi_{y}}{\partial M} \mathrm{~d} \chi_{y}+\frac{\partial M}{\partial a} \mathrm{~d} a$.

The term $\frac{\partial M}{\partial V} \mathrm{~d} V$ is zero, within the limits of our approximations, because the jolt $v$ is normal to the trajectory, so that the scalar variation $\mathrm{d} V$ is zero. Conversely, the reflected effect of the jolt on the pitch is expressed in the angular alteration $\varphi$ of the trajectory, as we noted in the beginning of this study: now, we can remark as a second approximation that $\tan \varphi \sim \varphi=\frac{v}{V}$ so that we have not $\delta \chi_{y}=n \theta$ but $\delta \chi_{y}=n(\theta-\varphi)$ and it results a second pitch oscillation $I \frac{\mathrm{d}^{2} \theta_{2}}{\mathrm{~d} t^{2}}=C \varphi=\frac{C}{V} v$, where $C$ and the subsequent $C_{1}$ and $C_{2}$ are constants to be easily determined. Recalling the expression of $v$, it immediately results:

$\theta_{2}=C_{1} t^{2}+C_{2} e^{-\mu t} \cos (h t-3 \psi)$.

The non-zero limit value of the jolt speed, i.e. the constant angular variation of the trajectory, would therefore produce a $\theta_{2}$ increasing with time, that is an intrinsic instability of the aircraft; but apart from the approximations and simplifying hypotheses from which this conclusion derives, the fact always remains that in reality it tends to zero as a result of the drags of the aircraft.

Publisher's Note Springer Nature remains neutral with regard to jurisdictional claims in published maps and institutional affiliations. 